\title{
TEM Based Micro-texture Measurement for Twinning in a Hot-rolled Magnesium Alloy with Astar System
}

\author{
Zhen Zhang, Edgar F. Rauch and Muriel Veron
}

SIMAP laboratory, CNRS-Grenoble INP, BP 46101 rue de la Physique, 38402 Saint Martin d'Hères, France.

Electron Backscatter Diffraction (EBSD), which is a scanning electron microscope (SEM) based technique to examine the microstructural-crystallographic information, may provide an effective way for micro-texture analysis [1]. This famous technique has attracted increasing interests and rapidly developed during the past few decades. It, however, has some inherent disadvantages, such as limited spatial resolution and its low efficiency for deformed materials. Indeed, EBSD technique relies on the indexing of Kikuchi patterns of backscattering electrons, whose quality gets deteriorated with the increase of local plastic strain. This renders its application on severely deformed metals almost impossible.

Besides, the application of EBSD technique on magnesium alloys itself faces specific challenges. As one of the lightest metals, the signal from backscatter electron is very low. This, in turn, requires very careful and tedious specimen preparation procedures. Moreover, in magnesium alloys strain localization usually accompanies twin formation, and this makes orientation indexing quite difficult in twinned area $[2]$.

In the present work, TEM based automatic orientation mapping system was used to characterize the micro-texture of twinning area in a hot-rolled $\mathrm{mg}$ alloy. With the series tilting scanning method, twinning type was completely identified and rigorous orientation analyses were carried out to investigate the twinning behavior in the hot rolling process of this alloy.

Automatic crystal orientation measurements were performed with ASTAR system on a JEOL 3010 TEM operated at $300 \mathrm{KV}$. Selected areas were scanned by electron beam in Nano-beam mode with a step size of $20 \mathrm{~nm}$ under the control of Digi star device developed by NanoMEGAS. During scanning, the diffraction patterns from each pixel was recorded and stored in the computer's memory. The orientation/phase identification was then performed off-line using a template matching algorithm. The so-called templates are a set of simulated electron diffraction patterns whose zone axes are covering the whole range of possible orientations with a given interval(less than $1^{\circ}$ ). By comparing the experimentally acquired diffraction pattern to the template, the best match is identified for each pixel and provides the local crystal orientation.

Figure 1 shows a series of Inverse Pole figure (IPF) maps for a single twin with different tilting angles ranging from $-15^{\circ}$ to $+15^{\circ}$ in an interval of $5^{\circ}$. The color code indicated the normal direction of the image plane, with respect to crystal coordinates in the orientation triangle. The twin was observed to have a $\langle\mathbf{1 1 2 0}\rangle-\mathbf{4 0}^{\circ}$ misorientation with matrix, which is close both to $\{\mathbf{1 0 1} 1\}-\{\mathbf{1 0 1}\}$ and $\{\mathbf{1 0 1 3}\}-\{\mathbf{1 0 1 2}\}$ double twinning. However, none of the $\left\{\mathbf{1 0 1}^{-1}\right\}$ plane trace coincides well with the shape of the twin, which excludes the possibility of the initial twinning event on any of the $\{10 \overline{1}\}$ planes. The (011i) plane trace, on the other hand, almost kept stationary during the tilting of specimen, and invariably coincided with the twin morphology in the tilting series image. This single twin could be thus defined as a $\{\mathbf{1 0 \overline { 1 }}\}-\{\mathbf{1 0} \overline{2}\}$ 
double twin. In plane trace analyses of normal EBSD, it is common that sometimes several crystallography planes are quite close to each other, and this ambiguity makes rigorous identification of twinning type impossible. With this tilting series method combined to ASTAR system, different crystallography planes can be always distinguished with each other from their traces, which makes rigorous orientation always available.

In normal TEM observation, certain zone axis is always essential for certain analyses, eg. high resolution image, double beam condition for defect analyses, etc. Finding certain zone axis is widely believed to be the most time consuming. The ASTAR system, however, provides a simple way to do this. Figure 2(a) shows an IPF map of twinning area. The $<11-20>$ pole figure was plotted for matrix (Figure 2(b)). By selecting a $<11-20>$ pole in the figure, it simply indicated the value for tilting $\mathrm{X}$ and $\mathrm{Y}$ of the specimen holder to make this zone axis parallel to the incident beam. By following the value provided $\left(T_{x}=7, T_{y}=10\right)$, the $<11-20>$ zone axis was acquired as shown in Figure 3(c).

\section{References:}

[1] Adam J. Schwartz, Mukul Kumar, Brent L Adams, Electron Backscatter Diffraction in Materials Science (Springe, Plenum)

[2] Z.Zhang, P. Cizek, M.Barnett, Scripta Materialia 67 (2012) 1015
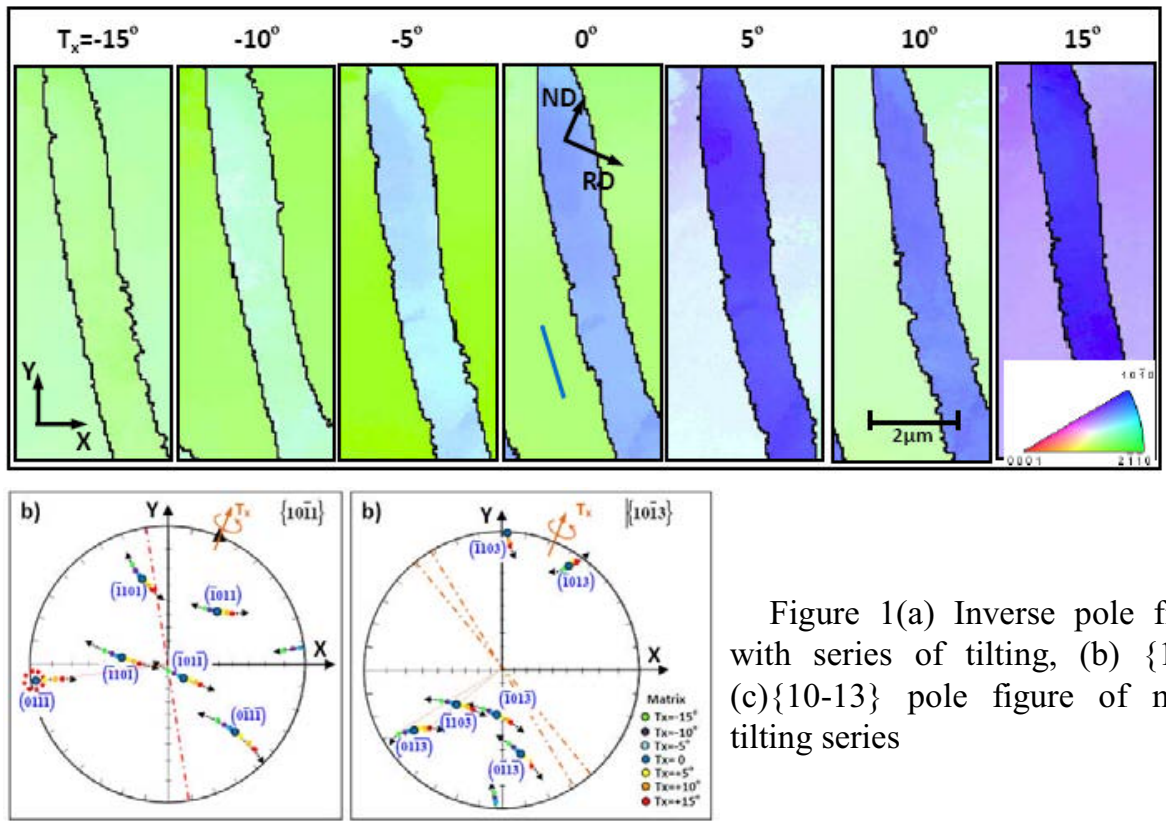

Figure 1(a) Inverse pole figure maps with series of tilting, (b) $\{10-11\}$ and (c) $\{10-13\}$ pole figure of matrix with tilting series
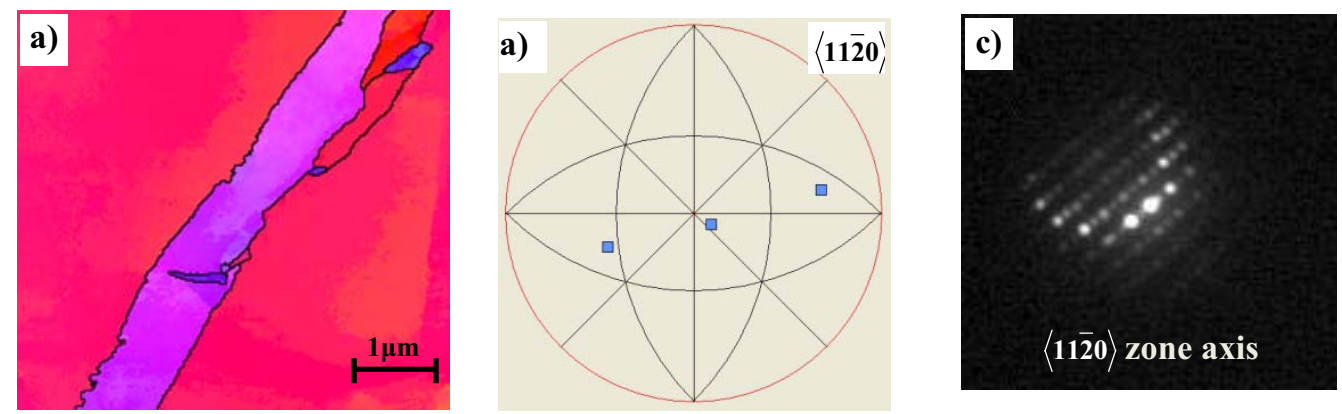

Figure 2(a) Inverse pole figure map of a single twin in magnesium alloy, (b) $<11-20>$ pole figures of matrix, (c) the diffraction pattern from $<11-20>$ zone axis in matrix 